ORTHODONTICS

\section{The influence of different facial components on facial aesthetics}

Faure JC, Rieffe C et al. Eur J Orthod 2002; 24: 1-7

Beauty is also in the eyes of the observed!

Facial aesthetics influence perception and behaviour. Photos of 36 orthodontic patients aged 11-17 yrs were assessed by 50 undergraduate students. Three versions were made of 11 photos: an original, one with inter-ocular distance enlarged by $20 \%$, and one with symmetry produced by mirroring half the face. These were incorporated into 3 series of photos. The panel was asked to score aethetics from 0-100 for each series of photos, compared with a reference photo given a score of 60 .

The 25 control photos were scored similarly at the three sessions (mean 46). The 11 experimental photos were scored a mean 44.4 in original form, $42.5(P<0.01)$ in symmetry and $38.6(P<0.001)$ with enlarged inter-ocular distance. The authors consider that if this degree of enlargement had been less, say $10 \%$, the result might have been more, rather than less aesthetic, and they note that other factors may also affect aesthetic perception.

\section{BEHAVIOURAL SCIENCE}

\section{Dental fear in sexually abused women}

Willumsen T Eur J Oral Sci 2001; 109: 291-296

Oral sexual abuse led to the greatest level of dental fear.

In Norway, there are regional support centres for sexually abused persons, and this study compared dental fear levels in 13 subjects reporting sexual touching (ST; mean age $44 \mathrm{yrs}$ ), 41 reporting intercourse (IC; 39 yrs) and 44 reporting oral penetration (OP; 35 yrs). More than half had experienced other forms of abuse, and more than half could not remember how many times they had been abused.

Fear was assessed by Corah's Dental Anxiety Scale: Norwegian norm for women is 8.5; for ST the mean was 12.1, for IC, 12.4 and for OP, 15.1. Respective scores for Kleinknecht's Dental Fear Scale were 2.2, 2.8, 3.0, 3.7. Scores for OP were significantly higher than for ST and IC, and 47\% of ST, 77\% of IC and 96\% of OP reported they had experienced problems in respect of dental treatment. The authors discuss how the dental problems raised by sexual abuse should be managed, and particularly note the severe vulnerability of these patients, some of whom do not realize the origin of their fear.
PERIODONTICS; ORAL SURGERY

\section{Mandibular second molar periodontal status after third molar extraction}

\section{Peng K-Y, Tseng Y-C et al. J Periodonto/ 2001; 72: 1647-1651}

Surgical removal of third molars is likely to cause attachment loss on second molars.

The study compared 58 second molars where the adjacent third molar had been surgically removed (42 subjects, mean age $48 \mathrm{yrs}$ ) more than 5 years ago with 20 controls where the third molar was congenitally absent (15 subjects, 55 yrs). Third molars had been removed 5-9 yrs before in 4/5 cases, and in others a longer time had elapsed. No subject had received periodontal care.

Mesial site probing depths were similar in control (mean 4.2 $\mathrm{mm}$ ) and experimental groups (4.2 $\mathrm{mm})$; distal control sites were shallower (5.7 v. $4.0 ; P<0.01)$. Attachment loss matched this (mesial 4.0 and 4.3 ; distal 4.1 and 5.8, $P<0.01$ ). Mean radiographic bone loss followed the same pattern. Recession was similar for all sites. The authors recommend periodontal follow-up when third molars are surgically removed.

\section{GERIATRIC DENTISTRY}

\section{Tooth loss in the very old: 13-15-year incidence among elderly Iowans}

Warren JJ, Watkins CA et al. Community Dent Oral Epidemio/ 2002; 30: 29-37 This long-term study suggests significant tooth loss in the 65+ age group.

Oral examination was performed for 520 subjects in 1983 and repeated up to 1998 when, of those who could be contacted, 73 subjects participated but 102 did not, largely for reasons of illness. These 2 groups were similar, except that non-participants had slightly more baseline attachment loss ( 2.2 v. $1.9 \mathrm{~mm}$ ). Mean follow-up was 14 yrs. Over that time, those initially aged 65-69 yrs reduced median tooth number from 24 to 21, those aged 70-74 from 22 to 18.5 and those aged 75-79, from 18.5 to 18 . Canines and maxillary incisors were least likely, and molars the most likely, to be lost.

When 153 lost teeth were compared with 1303 retained teeth, there were significant differences in mean DF coronal surfaces (1.25 v. 0.71), and attachment loss (based on a reduced number of teeth). Those who had difficulty eating, swallowing and smiling, or had oral discomfort or pain, had significantly fewer teeth. The authors noted that the accumulated tooth loss at this time of life was likely to affect well-being. 